\title{
Pérdidas de nitrógeno por volatilización a partir de dos fuentes nitrogenadas y dos métodos de aplicación
}

\author{
Nitrogen losses due to volatilization from two nitrogenous \\ sources and two application methods
}

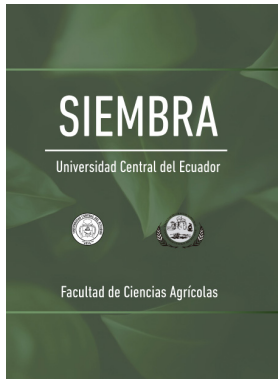

\author{
Roberto Carlos Larios-González ${ }^{1 *}$, Leonardo García Centeno², \\ Miguel Jerónimo Ríos ${ }^{3}$, Crysbell del Socorro Avalos Espinoza ${ }^{4}$, \\ Joseling Rubí Castro Salazar ${ }^{5}$
}

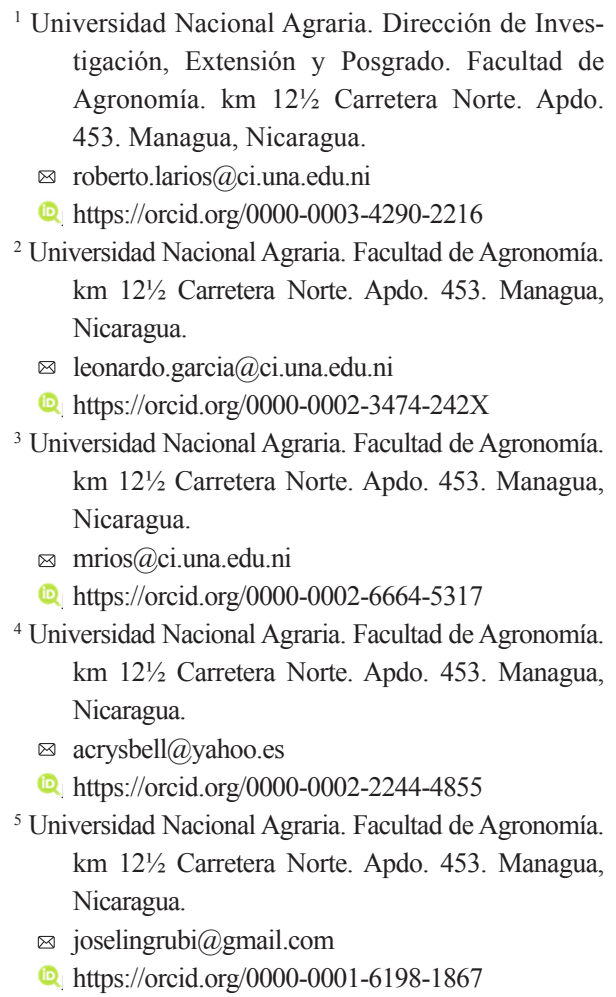

凶 leonardo.garcia@ci.una.edu.ni

${ }^{3}$ Universidad Nacional Agraria. Facultad de Agronomía.

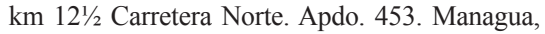
Nicaragua.

凶rios@ci.una.edu.ni

D. https://orcid.org/0000-0002-6664-5317

${ }^{4}$ Universidad Nacional Agraria. Facultad de Agronomía.

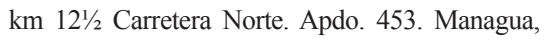
Nicaragua.

凶acrysbell@yahoo.es

D. https://orcid.org/0000-0002-2244-4855

${ }^{5}$ Universidad Nacional Agraria. Facultad de Agronomía.

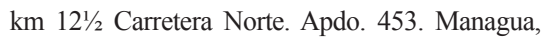
Nicaragua.

凶joselingrubi@gmail.com

D. https://orcid.org/0000-0001-6198-1867

*Autor de correspondencia: roberto.larios@ci.una.edu.ni

\section{SIEMBRA}

https://revistadigital.uce.edu.ec/index.php/SIEMBRA ISSN-e: $2477-8850$

ISSN: $1390-8928$

Periodicidad: semestral

vol. 8, núm. 2, 2021

siembra.fag@uce.edu.ec

DOI: https://doi.org/10.29166/siembra.v8i2.2475

Esta obra está bajo una licencia internacional Creative Commons Atribución-NoComercial

\section{Resumen}

La volatilización es un proceso más de pérdida del nitrógeno, puede aumentar según factores edafoclimáticos, fuente y método de aplicación. Este estudio se realizó en la finca El Plantel, propiedad de la Universidad Nacional Agraria, ubicada en Masaya, Nicaragua. El objetivo fue cuantificar las pérdidas de nitrógeno por volatilización y su influencia en el rendimiento del cultivo de sorgo (Sorghum bicolor L.). Se evaluaron dos fertilizantes nitrogenados y dos métodos de aplicación en un diseño de bloque completo al azar con cuatro tratamientos y tres repeticiones. Los tratamientos fueron: urea $46 \%$ aplicada en la superficie del suelo, urea incorporada al suelo, sulfato de amonio en la superficie y sulfato de amonio incorporado. La fertilización nitrogenada se realizó de manera fraccionada, $30 \%\left(57 \mathrm{~kg} \mathrm{~N} \mathrm{ha}^{-1}\right)$ al momento de la siembra y $70 \%$ (133 $\mathrm{kg} \mathrm{N} \mathrm{ha}^{-1}$ ) a los 35 días después de la siembra. Los muestreos de la volatilización se registraron los días uno, tres, seis y nueve después de la primera fertilización y el segundo en la misma secuencia a partir del segundo día después de la segunda fertilización. Se realizó un análisis de varianza y separación de medias por diferencias mínimas significativas de Fisher con $95 \%$ de confiabilidad usando InfoStat profesional 2009. Las pérdidas por volatilización son mayores cuando se aplica urea en la superficie del suelo $\left(57,68 \mathrm{~kg} \mathrm{~N} \mathrm{ha}^{-1}\right)$, lo que representa un 43,37 \%. Los rendimientos son iguales al incorporar las fuentes nitrogenadas.

Palabras clave: dinámica de nitrógeno, fertilización nitrogenada, sulfato de amonio, urea, sorgo.
Abstract
Nitrogen loss volatilization is one of the processes that may be greater depending on edaphic and climatic factors, it also depends on the source and the application method. This study was conducted at the farm El Plantel, owned by the Universidad Nacional Agraria, located at Masaya, Nicaragua. The objective of the study was to quantify the nitrogen losses due to volatilization and its influence on the yield of sorghum (Sorghum bicolor L.). Treatments consisted on two nitrogen fertilizers and two application methods. Treatments were as follow: $46 \%$ urea applied to the soil surface, urea incorporated into the soil, ammonium sulfate on the 
soil surface, and ammonium sulfate incorporated in soil. Nitrogen fertilization was carried out fractionally, $30 \%$ (57 kg N $\left.\mathrm{ha}^{-1}\right)$ at planting and $70 \%\left(133 \mathrm{~kg} \mathrm{~N} \mathrm{ha}^{-1}\right) 35$ days after planting. The four treatments were set up in a randomized complete block design with three replications. Volatilization losses samplings were recorded on day one, three, six and nine after the first fertilization, and the second sampling was carried out in the same sequence from the second day after the second fertilization. An analysis of variance and mean separation were performed by less significant differences of Fisher $95 \%$ using professional InfoStat 2009 programm. Volatilization losses are higher when urea is applied on the soil surface (57.68 $\mathrm{kg} \mathrm{N} \mathrm{ha}^{-1}$ ), reaching a cumulative loss of $43.37 \%$. Yields was statistically similar among the sources of nitrogen evaluated.

Keywords: nitrogen dynamics, nitrogen fertilization, ammonium sulfate, urea, sorghum.

\section{Introducción}

El nitrógeno, por su dinámica en el suelo, está sometido a perderse temporal o permanentemente. Salvagiotti y Vernizzi (2006) plantean que la fuente nitrogenada y el método de aplicación, son aspectos relevantes a considerar para lograr un uso eficiente del nitrógeno. Este elemento se puede perder por lixiviación, principalmente como nitrato $\left(\mathrm{NO}_{3}\right)$ (Navarro García y Navarro García, 2013), por volatilización en sus estados gaseosos como el amoníaco $\left(\mathrm{NH}_{3}\right)$ (González Osorio y Sadeghian Khalajabadi, 2012), también como óxido nitroso $\left(\mathrm{N}_{2} \mathrm{O}\right)$, óxido de nitrógeno (NO), dióxido de nitrógeno $\left(\mathrm{NO}_{2}\right.$ ) (Navarro García y Navarro García, 2013), o bien por absorción de las plantas, inmovilización de microorganismos (Navarro Blaya y Navarra García, 2003) y por erosión (Camas Gómez et al., 2012). Sus pérdidas están influenciadas por factores edáficos y climáticos; en el caso de la volatilización, el manejo se constituye en un importante factor. Según Núñez-Escobar (2016), también ocurren pérdidas por desnitrificación, que consiste en un proceso de reducción bioquímica del nitrato $\left(\mathrm{NO}_{3}\right)$ transformándose en $\mathrm{N}_{2} \mathrm{O}$, $\mathrm{NO}$ o hasta en su forma elemental $\left(\mathrm{N}_{2}\right)$.

La volatilización de nitrógeno en forma de amoníaco es un proceso normal en los suelos producto de la mineralización del nitrógeno orgánico, sin embargo, las pérdidas por volatilización son mayores, según Chevallier Boutell y Toribio (2006), cuando provienen de fertilizantes químicos. Las pérdidas por este proceso pueden alcanzar, dependiendo de la fuente, valores entre $3 \%$ y $55 \%$ (Hargrove et al., 1977, como se citó en Núñez-Escobar, 2016); favorecidos por procesos químicos, físicos y biológicos; afectados por el clima, el suelo, el manejo, la temperatura, el pH, la capacidad de intercambio catiónico, el contenido de materia orgánica, la cobertura del suelo, la calidad de residuos, así como las dosis y localización del fertilizante (Ferraris et al., 2009).

El nitrógeno es un elemento esencial para el crecimiento y desarrollo de los cultivos, por lo que es relevante en los sistemas de producción agrarios; Demolón (1995) indica que este elemento se relaciona de manera destacada con el crecimiento de las plantas, debido a la producción y síntesis de aminoácidos, los que fungen como componentes básicos de proteínas, enzimas y vitaminas.

En Nicaragua no se registran estudios sobre pérdidas de nitrógeno por volatilización, por lo que se decidió realizar esta investigación en un sistema de producción de sorgo. Este cultivo es considerado un grano básico debido a su importancia y uso en la producción de alimento humano y animal. El objetivo de esta investigación fue cuantificar las pérdidas por volatilización en función de dos fuentes nitrogenadas — urea al 46 \% y sulfato de amonio (21\% de nitrógeno y $24 \%$ de azufre)—, y dos métodos de aplicación: sobre la superficie del suelo e incorporado al suelo a aproximadamente $10 \mathrm{~cm}$ de profundidad.

\section{Materiales y métodos}

\subsection{Localización y descripción del área de estudio}

El estudio se realizó de noviembre del 2015 a febrero del 2016 en la Unidad de Investigación y Validación El Plantel, propiedad de la Universidad Nacional Agraria, finca localizada en el kilómetro 30 de la carretera Tipitapa-Masaya, departamento de Masaya, Nicaragua, en las coordenadas $12^{\circ} 06$ '23" de latitud norte y $86^{\circ} 05^{\prime} 37^{\prime}$ 'de longitud oeste, a 120 metros sobre el nivel del mar. En esta zona las lluvias por año varían de 600 a 1.800 milímetros; se registra una temperatura media anual de $28{ }^{\circ} \mathrm{C}$ y humedad relativa promedio de $70 \%$. 


\subsection{Condiciones edáficas}

La clase textural del suelo de la finca El Plantel se clasifica como franco arcilloso, con pH muy ligeramente ácido $(6,23)$, presenta niveles medios de materia orgánica $(2,62 \%)$ y nitrógeno $(0,13 \%)$; son suelos bajos en fósforo $(4,81 \mathrm{ppm}) \mathrm{y}$ altos en potasio $(1,34)$, calcio $(25,17) \mathrm{y}$ magnesio $(6,49 \mathrm{meq} / 100 \mathrm{~g}$ suelo).

\subsection{Descripción de los tratamientos}

Se evaluaron dos factores: el factor A corresponde a dos fuentes nitrogenadas, urea (46\%) y sulfato de amonio - $\left(\mathrm{NH}_{4}\right)_{2} \mathrm{SO}_{4}$ - y el factor $\mathrm{B}$, a dos métodos de aplicación, sobre la superficie del suelo e incorporado a aproximadamente $10 \mathrm{~cm}$ de profundidad. Se estableció una quinta parcela (testigo) a la que no se le aplicó fertilizante, y su objetivo fue determinar la volatilización que ocurre del nitrógeno natural que existente en el suelo, y con ello restar esa cantidad al nitrógeno volatilizado en las parcelas fertilizadas para determinar la pérdida por volatilización proveniente de las fuentes nitrogenadas.

La fertilización nitrogenada se realizó de manera fraccionada aplicando el $30 \%\left(57 \mathrm{~kg} \mathrm{~N}\right.$ ha $\left.{ }^{-1}\right)$ el día de la siembra y el restante $70 \%$, que equivale a $133 \mathrm{~kg} \mathrm{~N} \mathrm{ha}^{-1}$ a los 35 días después de la siembra (dds). Se aplicaron $95 \mathrm{~kg} \mathrm{ha}^{-1}$ de fósforo y $100 \mathrm{~kg} \mathrm{ha}^{-1}$ de potasio, ambos al momento de la siembra.

\subsection{Diseño experimental}

Se utilizó un arreglo bifactorial en diseño de bloques completos al azar (BCA) con cuatro tratamientos y tres repeticiones. La dimensión de cada unidad experimental fue de cuatro metros de largo por cuatro metros de ancho $\left(16 \mathrm{~m}^{2}\right)$, con separación de un metro entre parcelas y bloques para un total de $266 \mathrm{~m}^{2}$ como área experimental. Se consideró como parcela útil los dos surcos centrales con longitud de dos metros, para un área de $1,2 \mathrm{~m}^{2}$.

\subsection{Variables evaluadas}

\subsubsection{Volatilización de nitrógeno $\left(\mathrm{kg} \mathrm{ha}^{-1}\right)$}

La volatilización del nitrógeno se evaluó en dos momentos: a la siembra y a los 35 días después de la siembra (dds); en el caso de la primera fertilización, se consideró únicamente la fuente nitrogenada (urea y sulfato de amonio), no así el método de aplicación, debido a que la fertilización inicial se realizó al momento de la siembra y al fondo del surco. Tanto la fuente y el método de aplicación fueron analizados en la segunda fertilización y se utilizó una parcela testigo para descontar, a las pérdidas desde los tratamientos, las pérdidas del suelo sin fertilizar, y así calcular las pérdidas efectivas desde las fuentes nitrogenadas. Para la cuantificación del nitrógeno volatilizado se utilizó la metodología de Araújo et al. (2009), que consiste en el uso de una cámara artesanal semiabierta estática captadora de amoníaco.

La cámara se construyó usando una botella plástica con capacidad de dos litros, cuyo fondo (base) fue retirado, dejando un área de captación igual a $0,008 \mathrm{~m}^{2}$, con el objetivo de permitir la circulación y captación del amoníaco en la cámara. La base de la botella fue colocada a manera de paraguas en la parte superior de la botella (Figura 1), como mecanismo de protección contra el agua de riego y lluvia. La protección de las cámaras era para evitar el lavado de la lámina de espuma de poliuretano impregnada con ácido sulfúrico (sustancia captadora de amoníaco), así como el derrame del recipiente que contiene y abastece constantemente de ácido sulfúrico por capilaridad a la lámina de espuma.

En el interior de la cámara se ubicó el sistema de captación de amoníaco, que consiste en una lámina de espuma de poliuretano de $2,5 \mathrm{~cm}$ de ancho y $25 \mathrm{~cm}$ de longitud; esta cinta es impregnada con ácido sulfúrico un molar; la lámina se ubica suspendida de manera vertical a lo largo del recipiente desde la parte superior hasta un depósito plástico ubicado a lo interno de la botella, el cual contiene el ácido sulfúrico (Figura 1). 


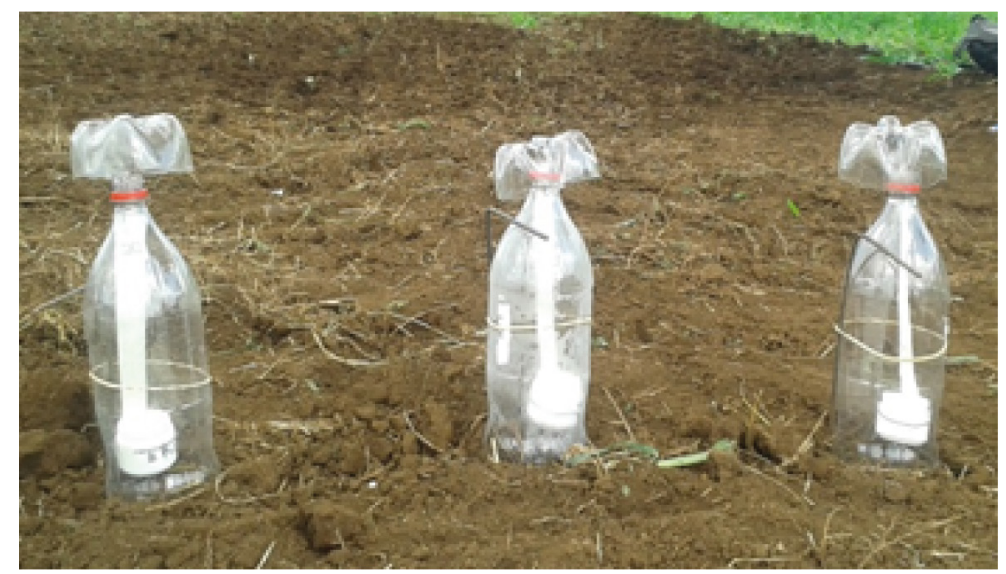

Figura 1. Cámara artesanal de captación de amoníaco.

Figure 1. Artisanal chamber for ammonia collection.

En cada parcela útil se fijaron con estacas al suelo, tres cámaras de captación; el muestreo (colecta de la lámina de poliuretano) se realizó un día después de la aplicación del fertilizante, luego al tercero, sexto y noveno día después de la fertilización, según la metodología de Araújo et al. (2009). Cada cámara de captación, luego del muestreo, fue ubicada en el mismo lugar en la parcela útil con una nueva lámina de espuma de poliuretano.

Debido a que el área del paraguas es de $0,008 \mathrm{~m}^{2}$, y que el movimiento del agua no solo ocurre de manera vertical, sino en diferentes direcciones por capilaridad (horizontal y diagonal), el nitrógeno se moviliza en el perfil del suelo, reduciendo con el tiempo las pérdidas por volatilización.

Las muestras se colectaron y se trasladaron al laboratorio de suelos y agua de la Universidad Nacional Agraria, en Managua, para la determinación del nitrógeno captado. La determinación del nitrógeno se realizó por el método de Kjeldhal mediante destilación, donde se libera el amoníaco, el que es retenido en una solución de ácido bórico y su determinación por titulación para así obtener la cantidad de amoníaco perdido y presente en las muestras destiladas.

Antes de realizar el análisis de las muestras, el equipo fue calibrado mediante pruebas de recuperación, obteniéndose un promedio de $98 \%$; para esto se utilizó el método Kjeldhal y se siguió el procedimiento establecido para pruebas de recuperación descrito por FOSS Application Note (2008).

\subsubsection{Rendimiento de grano $\left(\mathrm{kg} \mathrm{ha}^{-1}\right)$}

La cosecha del grano se realizó en los dos surcos centrales (parcela útil). El rendimiento obtenido se expresó en $\mathrm{kg} \mathrm{ha}^{-1}$ y se ajustó a $14 \%$ de humedad según la ecuación [1], propuesta por Giraldo et al. (2000) que indica que:

$$
P i(100-H i)=P f(100-H f) \therefore P f=P i(100-H i) /(100-H f)
$$

Donde: $P f=$ Peso final; $P i=$ Peso inicial, $H i=$ Humedad inicial, $H f=$ Humedad final.

\subsection{Análisis estadístico}

Para las variables rendimiento de grano, pérdidas por volatilización a partir de la segunda fertilización y pérdidas totales de nitrógeno, se realizó un análisis de varianza y separación de medias mediante diferencias mínimas significativas de Fisher (DMS) con $95 \%$ de confiabilidad. En el caso de las pérdidas por volatilización a partir de la primera y segunda fertilización, los resultados también se presentan de manera descriptiva usando gráficos de líneas.

\subsection{Fertilización y manejo agronómico del cultivo}

La preparación del suelo se realizó de manera mecanizada, realizando un pase de arado y dos pases de grada. La siembra fue de manera manual el 11 de noviembre del 2016, depositando la semilla a chorrillo ralo con una distancia entre hilera de 0,6 metros. Al momento de la siembra se fertilizó con la fórmula 12-30-10. Con esta fórmula se suministró todo el fósforo $\left(95 \mathrm{~kg} \mathrm{ha}^{-1}\right)$, parte del requerimiento de potasio $\left(100 \mathrm{~kg} \mathrm{ha}^{-1}\right)$, el que se 
complementó con cloruro de potasio y el $30 \%$ de la dosis de nitrógeno $\left(57 \mathrm{~kg} \mathrm{ha}^{-1}\right)$. A los 35 dds se aplicó el 70 $\%$ restante del nitrógeno correspondiente a $133 \mathrm{~kg} \mathrm{~N} \mathrm{ha}^{-1}$ usando urea y sulfato de amonio según los tratamientos.

La lámina de riego aplicada fue de ocho milímetros con intervalo de dos días, para un total de 46 riegos equivalentes a $368 \mathrm{~mm}$ durante el ciclo del cultivo. Se realizó un raleo manual a los 20 dds con el fin de mantener una densidad poblacional adecuada. El manejo de arvenses fue con azadón y no se requirió de aplicaciones para el manejo de plagas y enfermedades. La cosecha se realizó manualmente al cumplirse el ciclo biológico del cultivo.

\section{Resultados y discusión}

\subsection{Volatilización de nitrógeno a partir de la siembra}

En la Figura 2 se observan las pérdidas de nitrógeno después de la primera aplicación de las fuentes nitrogenadas al momento de la siembra $\left(57 \mathrm{~kg} \mathrm{~N} \mathrm{ha}^{-1}\right)$. En este momento solo se evalúa la fuente, ya que el fertilizante se aplicó al fondo del surco; que es el método tradicional utilizado en cultivos de granos. El día cero corresponde al día de la siembra y la fertilización, y los resultados se presentan de manera descriptiva.

A partir del primer día después de la siembra, se registran pérdidas por volatilización, pero se alcanza una mayor volatilización al tercer día después de la siembra en el caso de la urea, y al sexto día para el sulfato de amonio. A partir de estos momentos, las pérdidas por volatilización se detienen. Esto nos permite reafirmar que los días de muestreo, de acuerdo con la metodología de Araújo et al. (2009), es correcta, es decir, no tiene caso aumentar los días de muestreo, pues las pérdidas de nitrógeno por volatilización ya no se registran. En la Tabla 1 se aprecia que el mayor porcentaje de pérdida de nitrógeno se alcanza cuando se utiliza urea.

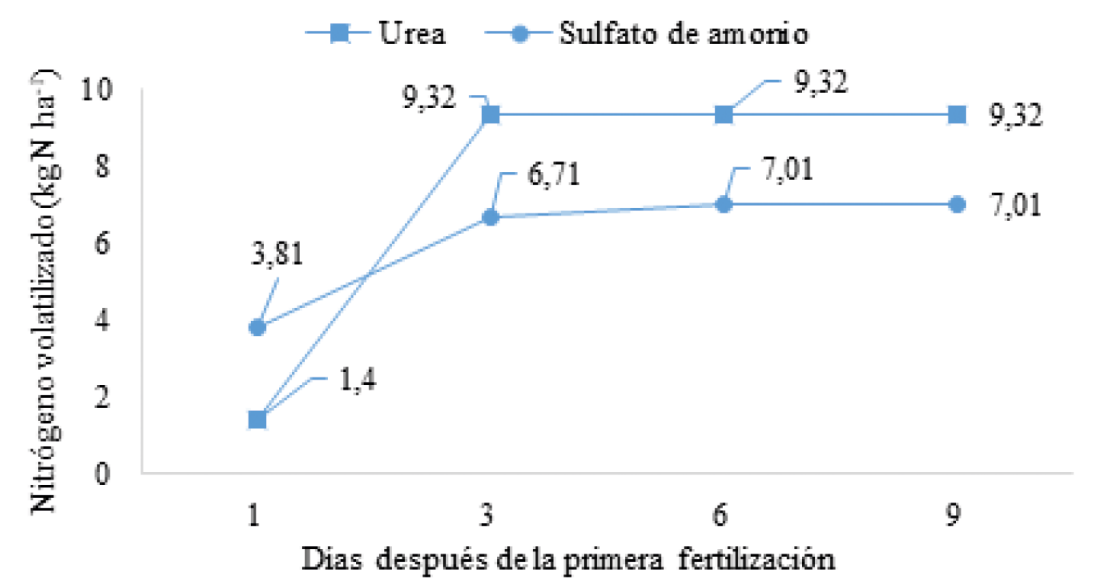

Figura 2. Pérdida de nitrógeno por volatilización $\left(\mathrm{kg} \mathrm{ha}^{-1}\right)$ aplicado al momento de la siembra. Figure 2. Nitrogen losses by volatilization $\left(\mathrm{kg} \mathrm{h}^{-1}\right)$ at sowing time.

Tabla 1. Pérdidas de nitrógeno (\%) según fuente nitrogenada y momento de muestreo a partir de la primera fertilización. Table 1. Nitrogen losses (\%) by nitrogen supply and time of sampling from the first fertilization.

\begin{tabular}{cccc}
\hline \multirow{2}{*}{$\begin{array}{c}\text { Fuente } \\
\text { nitrogenada }\end{array}$} & $\begin{array}{c}\text { Dosis de nitrógeno } \\
\text { kg N } \text { ha }^{-1}\end{array}$ & \multicolumn{2}{c}{ Pérdida (\%) de N por dds } \\
\cline { 3 - 4 } Urea $(46 \%)$ & 57,00 & Día 1 & Día 9 \\
Sulfato de amonio & 57,00 & 2,46 & 16,35 \\
\hline
\end{tabular}

dds: días después de la siembra. 
La mayor pérdida de nitrógeno proveniente de la urea ocurre debido a que al hidrolizarse se produce la formación de amoníaco $\left(\mathrm{NH}_{3}\right)$, forma química gaseosa que se volatiliza y que también alcaliniza el medio; situación que, combinada con las altas temperaturas, aumenta el proceso de pérdida, como lo indican Salvagiott y Vernizzi (2006). Las pérdidas provenientes de la urea y el sulfato de amonio en este estudio, coinciden con las reportadas por Schwenke (2014), quien señala que las pérdidas por volatilización son mínimas cuando las fuentes nitrogenadas son incorporadas al suelo. Pérdidas entre $12 \%$ y $20 \%$ por volatilización son reportadas por Hauck (1981); en este estudio los valores son inferiores a este rango. Otros resultados fueron registrados por Ferraris et al. (2009), quienes captaron emisiones de amoniaco desde el inicio del experimento, pero con diferencias estadísticas a partir del tercer día.

En este estudio, las pérdidas de nitrógeno se registran desde el primer día de muestreo ( 24 horas después de la aplicación), con mayor cantidad de nitrógeno volatilizado proveniente de la urea. El comportamiento de la temperatura a partir de la fertilización hasta nueve días después de la siembra (período de muestreo), se situó entre $32{ }^{\circ} \mathrm{C}$ y $34{ }^{\circ} \mathrm{C}$, condición que favorece la formación de amoníaco cuando las condiciones de humedad en el suelo son bajas.

\subsection{Volatilización de nitrógeno aplicado a los 35 dds}

A los 35 días después de la siembra se realizó la segunda fertilización nitrogenada ( $\left.133 \mathrm{~kg} \mathrm{~N} \mathrm{ha}^{-1}\right)$ de acuerdo con los métodos de aplicación (en la superficie e incorporado al suelo). El análisis estadístico indica que a las 24 horas después de la aplicación de las fuentes nitrogenadas, no se registran diferencias $(p=0,6266)$.

En la Figura 3 se observan las pérdidas de nitrógeno en función de las fuentes, método de aplicación (US: urea en superficie, UE: urea enterrada, SS: sulfato de amonio en superficie, SE: sulfato de amonio enterrado) y momento de muestreo, las que varían (al día nueve) desde 2,39 $\mathrm{kg} \mathrm{ha}^{-1}$ a 57,68 $\mathrm{kg} \mathrm{ha}^{-1}$ siendo mayores cuando se fertiliza con urea, principalmente al aplicarla en la superficie del suelo. Esto coincide con Sangoi et al. (2003), quienes reportan pérdidas mayores por volatilización cuando el fertilizante nitrogenado no es incorporado al suelo, y aumentan, como lo indican Salvagiott y Vernizzi (2006), cuando las temperaturas son altas.

Durante los nueve días de muestreo posterior a la segunda fertilización, las temperaturas aumentaron con relación al período del primer muestreo, oscilando entre $33{ }^{\circ} \mathrm{C}$ y $35^{\circ} \mathrm{C}$, condición que favoreció el proceso de volatilización. Igualmente, en este período, la cantidad de muestreos se realizó según la metodología de Araújo et al. (2009), quien indica un tiempo de registro de la volatilización de nueve días.

Estadísticamente los resultados indican que a partir del tercer día luego de la fertilización, el sulfato de amonio, indistintamente del método de aplicación, resulta en menor pérdida de nitrógeno por volatilización con respecto a la urea, ya sea aplicado en superficie o enterrado. También se registra al tercer y sexto día después de la fertilización, que la urea aplicada en la superficie del suelo es estadísticamente diferente a la urea enterrada. Esto es posible debido a que, al hidrolizarse la urea, la enzima ureasa provoca la formación de carbonato de amonio que rápidamente se descompone y libera amoníaco $\left(\mathrm{NH}_{3}\right)$. En la Tabla 2 se observan las pérdidas acumuladas en porcentaje, relacionadas con las pérdidas acumuladas de nitrógeno en $\mathrm{kg} \mathrm{ha}^{-1}$, variando desde $1,8 \%$ hasta $43,37 \%$.

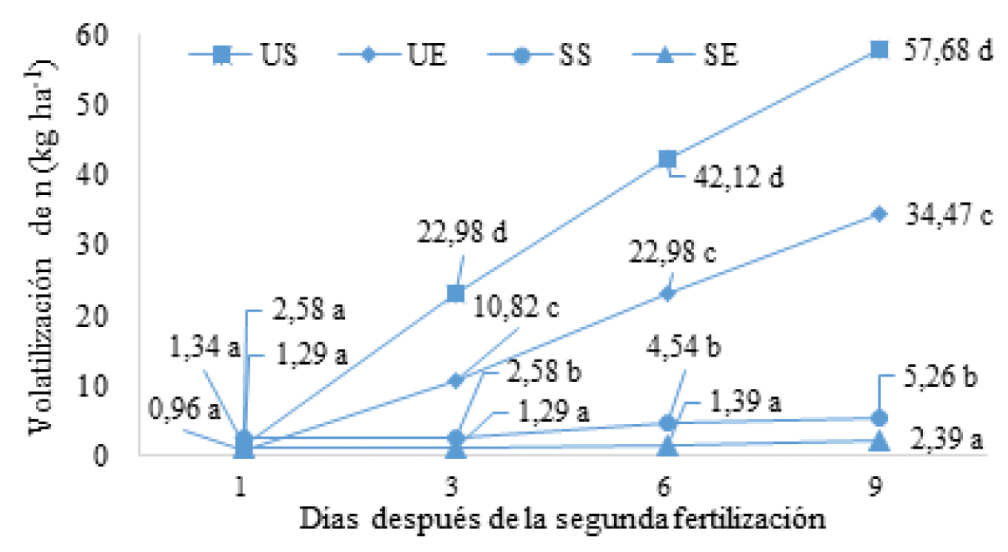

Figura 3. Pérdida de nitrógeno por volatilización a los 35 días después de la siembra (US: urea en la superficie, UE: urea enterrada, SS: sulfato de amonio en la superficie, SE: sulfato de amonio enterrado).

Figure 3. Nitrogen losses by volatilization at 35 days after sowing (US: surface urea, EU: buried urea, SS: surface ammonium sulfate, SE: buried ammonium sulfate). 
Tabla 2. Pérdidas de nitrógeno $\left(\mathrm{kg} \mathrm{ha}^{-1} \mathrm{y} \%\right)$ según fuente y método de aplicación a partir de la segunda fertilización. Table 2. Nitrogen losses $\left(\mathrm{kg} \mathrm{ha}^{-1}\right.$ and \%) by source and application method from the second fertilization.

\begin{tabular}{lcc}
\hline \multirow{2}{*}{ Fuente y método } & \multicolumn{2}{c}{ Pérdida } \\
\cline { 2 - 3 } & kg N ha $\mathbf{~}^{-1}$ & $\mathbf{\% ~ N}$ \\
\hline Urea en superficie & $57,68 \mathrm{c}$ & 43,37 \\
Urea enterrada & $34,47 \mathrm{~b}$ & 25,95 \\
Sulfato de amonio en superficie & $5,26 \mathrm{a}$ & 3,95 \\
Sulfato de amonio enterrado & $2,39 \mathrm{a}$ & 1,80 \\
\hline \multicolumn{1}{c}{ DMS } & 0,749 \\
\hline
\end{tabular}

dds: días después de la siembra.

Dominghetti et al. (2016) reportan $12 \%$ de pérdida de nitrógeno por volatilización al tercer día después de la primera fertilización con urea, y un acumulado de alrededor del $27 \%$ a los 10 días después de la aplicación, sus datos varían entre $19 \%$ y $21 \%$ con respecto a la segunda fertilización en el mismo período de tiempo; además, plantean que la eficiencia de la urea es menor y puede tener un impacto negativo en los costos de producción en comparación al sulfato de amonio.

Barrios et al. (2012) establecen que las condiciones de humedad afectan la solubilidad de la cubierta del grano de urea, aumentando las pérdidas por volatilización y disminuyendo la efectividad, sin embargo, el uso de sulfato de amonio en dosis bajas permite mayor disponibilidad de nitrógeno para la planta, por la eficiencia y resistencia del nitrógeno amoniacal a las pérdidas por volatilización y lixiviación.

González Osorio y Sadeghian Khalajabadi (2012) registraron pérdidas por volatilización de 3,4 \% y 8,1 $\%$ en los primeros dos días de fertilización utilizando urea, estableciendo que, en el proceso de hidrólisis, la producción de ureasa, genera un ambiente alcalino; en contraste con el sulfato de amonio, con el que la volatilización fue casi nula (1,3\%), debido a su composición amoniacal y al ambiente ácido que genera su hidrólisis.

Para ampliar la eficiencia de los fertilizantes es importante considerar los procesos de ganancia, pérdidas y transformación de los nutrientes, valorando los componentes físicos, químicos y biológicos del suelo, método y momento de aplicación. Morales-Morales et al. (2019) reportan volatilización de amoníaco a partir de urea de hasta $30 \mathrm{~kg} \mathrm{~N} \mathrm{ha}^{-1}(25 \%)$ y señalan que la transformación del nitrógeno a la forma gaseosa de $\mathrm{NH}_{3}$, es una vía de dispersión de nitrógeno en fertilizantes a base de urea cuando son aplicados en la superficie del suelo.

\subsection{Volatilización acumulada total}

El análisis de varianza no indica diferencias por efecto de la interacción de los factores en estudio, por lo que el análisis de éstos se hace de manera independiente. En la Figura 4 se presentan las pérdidas acumuladas de nitrógeno y se observa que la volatilización es mayor cuando se fertiliza con urea sobre la superficie del suelo, alcanzando pérdidas de $67 \mathrm{~kg} \mathrm{~N} \mathrm{ha}^{-1}(35,26 \%)$. Al comparar los valores de la volatilización cuando la urea es incorporada al suelo, las pérdidas son de $43,79 \mathrm{~kg} \mathrm{~N} \mathrm{ha}^{-1}(23,05 \%)$, lo que representa un $65,36 \%$ menos de pérdidas.

En el caso del sulfato de amonio, las cantidades y la diferencia en pérdidas en cuanto al método de aplicación son mucho menores; se registra $12,27 \mathrm{~kg} \mathrm{~N}^{-1}(6,46 \%)$ cuando se aplica en la superficie y 9,40 $\mathrm{kg}$ $\mathrm{N} \mathrm{ha}^{-1}$ (4,95\%) cuando es incorporado. Esto se debe en parte a lo que explica Lupi (2001), indicando que los protones liberados son mayores durante la transformación del $\left(\mathrm{NH}_{4}\right)_{2} \mathrm{SO}_{4}$ con respecto a la cantidad de protones liberados de la urea; además, señala que por cada mol de sulfato de amonio, se liberan cuatro unidades (moles) de $\mathrm{H}^{+}$, mientras que cada unidad mol de urea produce solo dos moles de $\mathrm{H}^{+}$; lo que significa que la acidez inducida por la nitrificación del sulfato de amonio es mayor a la acidez producida por la urea, condición que desfavorece la volatilización. 


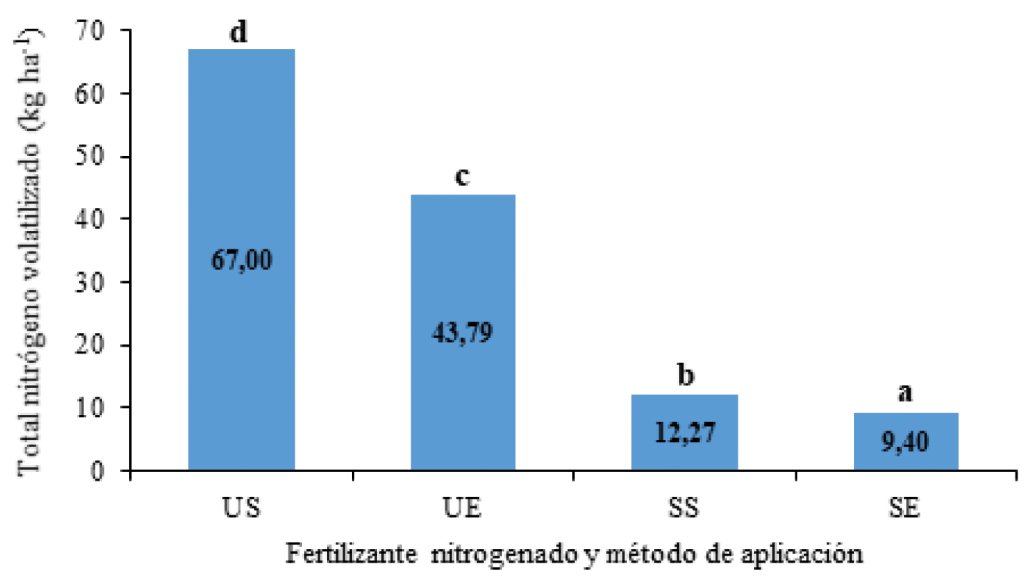

Figura 4. Pérdidas acumuladas de nitrógeno por volatilización según fuente y método de aplicación (US: urea en la superficie, UE: urea enterrada, SS: sulfato de amonio en la superficie, SE: sulfato de amonio enterrado).

Figure 4. Accumulated nitrogen losses by volatilization by source and application method (US: surface urea, EU: buried urea, SS: surface ammonium sulfate, SE: buried ammonium sulfate).

La mayor pérdida por volatilización de nitrógeno proveniente de la urea coincide con investigaciones realizadas por Jadon et al. (2018) quienes registraron un acumulado de 32,9\% de pérdidas en un período de 10 días, siendo mayor que otros fertilizantes nitrogenados evaluados en su estudio; al igual que Costa et al. (2003), que obtuvieron pérdidas de hasta $36 \%$. Se reportan múltiples diferencias en relación con las cantidades de nitrógeno que se pierden por volatilización, debido en parte a las características del fertilizante, método de aplicación, clima, así como a condiciones físicas, químicas y biológicas del suelo. Así lo han registrado diferentes autores al reportar pérdidas variables de nitrógeno, por ejemplo, Leal-Varón et al. (2007) encontraron pérdidas entre 30 \% y $35 \%$; Ordóñez et al. (2005) señalan 15,2 \%; mientras que Lara Cabezaas et al. (1997) obtuvieron pérdidas de hasta $50 \%$.

\subsection{Rendimiento del grano $\left(\mathrm{kg} \mathrm{ha}^{-1}\right)$}

No se registra efecto por la interacción de los factores. En la Tabla 3 se observa que, independientemente de la fuente nitrogenada, los rendimientos son mayores cuando el fertilizante se aplica por debajo de la superficie del suelo. Resultados iguales fueron reportados por Fontanetto et al. (2006), quienes obtuvieron mayores rendimientos cuando los fertilizantes nitrogenados se incorporaron al suelo, acción que aumenta el uso eficiente de los fertilizantes nitrogenados con beneficios adicionales, como los que plantea Trenkel (2010) al señalar que la reducción de las pérdidas de nitrógeno reducen el impacto negativo sobre el ambiente, disminuyen los costos de producción y se obtienen mayores rendimientos de manera consistente.

Tabla 3. Rendimiento $\left(\mathrm{kg} \mathrm{ha}^{-1}\right)$ según tratamiento.

Table 3. Yield $\left(\mathrm{kg} \mathrm{ha}^{-1}\right)$ by treatment.

\begin{tabular}{|c|c|}
\hline Tratamiento & Rendimiento \\
\hline Sulfato de amonio enterrado & $5.225 \mathrm{a}$ \\
\hline Urea (46 \%) enterrada & $5.089 \mathrm{a}$ \\
\hline Urea $(46 \%)$ en la superficie & $4.155 \mathrm{~b}$ \\
\hline Sulfato de amonio en la superficie & $3.345 \mathrm{~b}$ \\
\hline DMS & 836,82 \\
\hline
\end{tabular}

También se observa en la Tabla 3 que, los rendimientos con el uso de sulfato de amonio en superficie son estadísticamente iguales a los obtenidos con la aplicación de urea en la superficie, aunque las pérdidas prove- 
nientes del sulfato de amonio sean menores. Esta igualdad estadística respecto a la fuente y método de aplicación (sulfato de amonio enterrado, urea enterrada, urea en la superficie, sulfato de amonio en la superficie), se explica debido a que se utilizó una alta dosis de nitrógeno $\left(190 \mathrm{~kg} \mathrm{~N} \mathrm{ha}^{-1}\right)$ que permite este comportamiento, debido al aporte de nitrógeno de ambas fuentes después de las perdidas por volatilización. Sin embargo, indistintamente de la fuente, los fertilizantes nitrogenados deben ser incorporados al suelo. Grijalva-Contreras et al. (2016) mencionan que el uso de fertilizantes nitrogenados es esencial para optimizar el rendimiento debido a la importante función que el nitrógeno desempeña en los cultivos, y que su manejo eficiente posibilita reducir costos de producción.

Si los componentes de la ecuación de producción $(\mathrm{P}=[\mathrm{G}+\mathrm{S}+\mathrm{C}+\mathrm{M}]$, donde $\mathrm{G}=$ genética, $\mathrm{S}=$ suelo, $\mathrm{C}=$ clima y $\mathrm{M}=$ manejo), se presentan de forma favorable, los cultivos expresan su potencial de rendimiento. En el caso de la nutrición vegetal el nitrógeno es el elemento esencial responsable en gran parte de un mayor rendimiento. Compton (1990) afirma que el rendimiento del sorgo tiene una relación directa con la eficiencia de las plantas en la utilización de los recursos.

Sosa Rodríguez et al. (2019) mencionan que el nitrógeno es el nutriente más importante en la producción, siendo las principales fuentes, la mineralización de su fracción orgánica en el suelo y la adición en formas sintéticas y orgánicas. Barbieri et al. (2018) establecen que la eficiencia del uso del nitrógeno tiende a ser baja por la dinámica de este elemento en el suelo y por los procesos de transformación que incluyen pérdidas y ganancias.

Barrios et al. (2012), encontraron que el sulfato de amonio permite mayor disponibilidad de nitrógeno y más resistencia a la volatilización, lixiviación y desnitrificación, y que el uso de urea convencional ocasiona importantes pérdidas por volatilización.

En relación con la química del suelo, la volatilización es un proceso que es favorecido por el $\mathrm{pH}$ y la CIC (Ferraris et al., 2009); los suelos donde se realizó el experimento presentan valores de pH cercanos a la neutralidad $(6,23)$, valor que no aumenta la volatilización; y podemos inferir que por los niveles medios de materia orgánica $(2,62 \%)$, la capacidad de intercambio catiónica también es media, lo que podría favorecer que las pérdidas del amonio proveniente de la hidrólisis de la urea sean mayores, al no adsorberse al amonio en el complejo de cambio (Mikkelsen, 2010.); lo que no ocurre con el nitrógeno proveniente del sulfato de amonio, debido a que éste al solubilizarse, tiene un efecto acidificante en el suelo y disminuye el proceso de volatilización (Guerrero Riascos, 2004).

\section{Conclusiones}

Las pérdidas de nitrógeno por volatilización son considerablemente mayores cuando la fuente es urea, indistintamente del método de aplicación, lo que se traduce en una mayor pérdida acumulada de nitrógeno. La volatilización de nitrógeno proveniente de la urea aplicada en superficie es de 47,35 \% del total de nitrógeno aplicado en la segunda fertilización, siendo este el valor más alto en comparación con las pérdidas proveniente del sulfato de amonio, con ambos métodos de aplicación, y la urea enterrada.

El rendimiento del grano de sorgo es mayor cuando las fuentes nitrogenadas son incorporas al suelo, y aunque los rendimientos son menores cuando se fertiliza en la superficie del suelo, las fuentes tienen igual efecto en el rendimiento. En ambos casos, el uso de urea con mayores pérdidas por volatilización iguala al uso de sulfato de amonio, debido a la dosis alta de nitrógeno utilizada, que compensa la cantidad de nitrógeno volatilizado.

\section{Contribuciones de los autores}

- Roberto Carlos Larios González: conceptualización, análisis formal, administración de proyecto, recursos, investigación, visualización, redacción - revisión - edición.

- Leonardo García Centeno: conceptualización, análisis formal, investigación, visualización.

- $\quad$ Miguel Jerónimo Ríos: investigación, supervisor, visualización, redacción.

- Crysbell del Socorro Avalos Espinoza: análisis formal, investigación, metodología, visualización.

- Joseling Rubí Castro Salazar: análisis formal, investigación, metodología, visualización. 


\section{Referencias}

Araújo, E., da S.., Marsola, T., Miyazawa, M., Soares, L. H. de B., Urquiaga, S., Boddey, R. M., \& Alves, B. J. R. (2009). Calibração de câmara semiaberta estática para quantificação de amônia volatilizada do solo. Pesquisa Agropecuária Brasileira, 44(7), 769-776. https://doi.org/10.1590/S0100-204X2009000700018

Barbieri, P. A., Echeverría, H. E., \& Saínz Rozas, H. R. (2018). Pérdidas por volatilización y eficiencia de uso de nitrógeno en maíz en función de la fuente, dosis y momento de aplicación. Revista de la Facultad de Agronomía, 117(1), 111-116. https://revistas.unlp.edu.ar/revagro/article/view/7323

Barrios, M., García, J., \& Basso, C. (2012). Efecto de la fertilización nitrogenada sobre el contenido de nitrato y amonio en el suelo y la planta de maíz. Bioagro, 24(3), 213-220. http://ve.scielo.org/scielo.php?scrip$\mathrm{t}=$ sci_arttext\&pid=S1316-33612012000300007\&lng=es\&tlng=es

Camas Gómez, R., Turrent Fernández, A., Cortes Flores, J. I., Livera Muñóz, M., González Estrada, A., Villar Sánchez, B., López Martínez, J., Espinoza Paz, N., y Cadena Iñiguez, P. (2012). Erosión del suelo, escurrimiento y pérdida de nitrógeno y fósforo en laderas bajo diferentes sistemas de manejo en Chiapas, México. Revista Mexicana de Ciencias Agrícolas, 3(2), 231-243. http://www.scielo.org.mx/scielo. php?pid=S2007-09342012000200002\&script $=$ sci_arttext

Chevallier Boutell, S., \& Toribio, M. (2006). Volatilización del amoníaco. Información Técnica de Trigo. Instituto Nacional de Tecnología Agropecuaria, Estación Experimental Agropecuaria Rafaela. http://rafaela. inta.gov.ar/info/miscelaneas/105/trigo2006_69.pdf

Compton, L. P. (1990). Agronomía del sorgo. Programa de mejoramiento de sorgo del Instituto internacional para la investigación en cultivos para los trópicos semiáridos (ICRISAT).

Costa, M. C. G., Vitti, G. C., \& Cantarella, H. (2003). Volatilização de N-NH 3 de fontes nitrogenadas em cana-de-açúcar colhida sem despalha a fogo. Revista Brasileira de Ciência do Solo, 27(4), 631-637. https:// doi.org/10.1590/S0100-06832003000400007

Demolón, A. (1995). Principios de agronomía. Tomo II. Crecimiento de los vegetales cultivados. Pueblo y educación (4. ${ }^{\mathrm{a}}$ ed.). Ediciones Omega.

Dominghetti, A. W., Guelfi, D. R., Guimarães, R. J., Caputo, A. L. C., Spehar, C. R., \& Faquin, V. (2016). Nitrogen loss by volatilization of nitrogen fertilizers applied to coffee orchard. Ciência e Agrotecnologia, 40(2), 173-183. https://doi.org/10.1590/1413-70542016402029615

Ferraris, G. N., Couretot, L. A., \& Toribio, M. (2009). Pérdidas de nitrógeno por volatilización y su implicancia en el rendimiento del cultivo de maíz: efectos de fuente, dosis y uso de inhibidores. Informaciones Agronómicas, 43, 19-22. http://www.ipni.net/publication/ia-lacs.nsf/0/B5B2034B84BF8FF6852579950075F445/\$FILE/19.pdf

Fontanetto, H., Keller, O., Negro, C., \& Belotti, N. (2006). Pérdidas por volatilización de amoníaco de diferentes fuentes nitrogenadas en trigo bajo siembra directa. Instituto Nacional de Tecnología Agropecuaria, Estación Experimental Agropecuaria Rafaela. http://rafaela.inta.gov.ar/info/miscelaneas/105/ trigo2006_63.pdf

FOSS Application Note (2008). Rev. 8.0800 series unitadded, plus minor spelling corrections.

Giraldo, G., Méndez, M., \& Franco J. B. (2000). Manual para el manejo pre y poscosecha de semilla producida de manera artesanal bajo el esquema de pequeñas empresas de semillas. Centro Internacional de Agricultura Tropical [CIAT].

González Osorio, H., \& Sadeghian Khalajabadi, S. (2012). Volatilización del nitrógeno a partir de diferentes fuentes fertilizantes en la etapa de crecimiento vegetativo del café. Revista Cenicafé, 63(1), 132-143. https://biblioteca.cenicafe.org/jspui/bitstream/10778/518/1/arc063\%281\%29132-143.pdf

Grijalva-Contreras, R. L., Robles-Contreras, F., Macías-Duarte, R., Santillano-Cázares, J., \& Núñez-Ramírez, F. (2016). Nitrógeno en trigo y su efecto en el rendimiento y en la concentración de nitratos y potasio en el extracto celular de tallo (ECT). Acta Universitaria, 26(5), 48-54. http://dx.doi.org/10.15174/au.2016.963

Guerrero Riascos, R. (2004). Propiedades generales de los fertilizantes sólidos. Manual técnico. Monómeros Colombo Venezolanos S.A. http://www.monomeros.com/descargas/dpmanualfertilizacion.pdf

Hauck, R. D. 1981. Nitrogen fertilizer effects in nitrogen cycle processes. Ecological Bulletins, 33, 551-562. http://www.jstor.org/stable/45128688

Jadon, P., Selladurai, R., Yadav, S. S., Coumar, M. V., Dotaniya, M. L., Singh, A. K., Bhadouriya, J., \& Kundu, S. (2018). Volatilization and leaching losses of nitrogen from different coated urea fertilizers. Journal of soil science and plant nutrition, 18(4), 1036-1047. https://dx.doi.org/10.4067/S0718-95162018005002903 
Lara Cabezas, W. A. R., Korndorfer, G. H., \& Motta, S. A. (1997). Volatilização de N-NH 3 na cultura de milho: I. efeito da irrigação e substituição parcial da uréia por sulfato de amônio. Revista Brasileira de Ciência do Solo, 21(3), 481-487. https://doi.org/10.1590/S0100-06831997000300018

Leal-Varón, L. A., Salamanca-Jiménez, A., \& Sadeghian-Khalajabadi, S. (2007). Pérdidas de nitrógeno por volatilización en cafetales en etapa productiva. Revista Cenicafé, 58(3), 216-226. https://www.cenicafe. org/es/publications/arc058\%2803\%29216-226.pdf

Lupi, A. M. (2001). Efecto de diferentes fuentes de nitrógeno amoniacal sobre la acidificación del suelo. International Fertilizer Development Center.

Mikkelsen, R. (2010). Emisiones de amoníaco de operaciones agrícolas. Informaciones Agronómicas de Hispanoamérica (Northern Latin America, Mexico and Central America), 79, 24-27. http://www. ipni.net/publication/ia-lahp.nsf/0/251EB81E3731729F852579A0006A0E4E/\$FILE/Emisiones\%20 de\%20amoniaco.pdf

Morales-Morales, E. J., Rubí-Arriaga, M., López-Sandoval, J. A., Martínez-Campos, A. R., \& Morales-Rosales, E. J. (2019). Urea (NBPT) una alternativa en la fertilización nitrogenada de cultivos anuales. Revista Mexicana de Ciencias Agrícolas, 10(8), 1875-1886. https://doi.org/10.29312/remexca.v10i8.1732

Navarro Blaya, S., \& Navarro García, G. (2003). Química agrícola: El suelo y los elementos químicos esenciales para la vida vegetal. Mundi-Prensa.

Navarro García, G., \& Navarro García, S. (2013). Química Agrícola: química del suelo y de los nutrientes esenciales para para las plantas. Mundi-Prensa.

Núñez-Escobar, R. (2016). El suelo como medio natural en la nutrición de los cultivos. En G. Alcántar-González., L. I. Trejo-Téllez., y F. C. Gómez-Merino (eds.). Nutrición de cultivos (pp. 104-149). Colegio de postgraduados.

Ordóñez, S., Quintero, R., Larrahondo, E., \& Jaramillo, A. (2005). Evaluación de dos métodos analíticos para cuantificar las pérdidas de nitrógeno por volatilización en suelos del valle del Río Cauca. Carta Trimestral Cenicaña, 27(2), 4-9.

Salvagiotti, F., \& Vernizzi, A. (2006). Pérdidas de nitrógeno por volatilización y su efecto en el rendimiento del cultivo de maiz. Para mejorar la producción 32. Instituto Nacional de Tecnología Agropecuaria, Estación Experimental Agropecuaria Oliveros. http://anterior.inta.gov.ar/oliveros/info/documentos/ maiz/nutricion2.pdf

Sangoi, L., Ernani, P. R., Lech, V. A., \& Rampazzo, C. (2003). Volatilization of N-NH influenced by urea application forms, residue management and soil type in lab conditions. Ciência Rural, 33(4), 687-692. https://doi.org/10.1590/S0103-84782003000400016

Schwenke, G. (2014). Nitrogen volatilisation: factors affecting how much $\mathrm{N}$ is lost and how much is left over time. Grains Research and Development Corporation. https://grdc.com.au/resources-and-publications/ grdc-update-papers/tab-content/grdc-update-papers/2014/07/factors-affecting-how-much-n-is-lost-andhow-much-is-left-over-time

Sosa Rodríguez, B. A., Sánchez de Prager, M., García Vivas, Y. S., Espinoza Guardiola, M. D., Rodríguez, J. A., \& Sosa Rodríguez, G. M. (2019). Dinámica de nitrógeno del suelo en agroecosistemas bajo el efecto de abonos verdes. Acta agronómica, 68(4), 257-264. https://doi.org/10.15446/acag.v68n4.71963

Trenkel, M. E. (2010). Slow -and controlled-release and stabilized fertilizers: an option for enhancing nutrient use efficiency in agriculture. International Fertilizer Industry Association (IFA). https://www.fertilizer.org/images/Library_Downloads/2010_Trenkel_slow\%20release\%20book.pdf 